\title{
DAS MATHEMATISCHE SCHAFFEN ROLF NEVANLINNAS
}

\author{
LARS AHLFORS *
}

Ich habe den ehrenvollen Auftrag bekommen, bei dieser Veranstaltung den Eröffnungsvortrag zu halten. Nichts könnte mir eine grössere Freude bereiten, als unseren Ehrengast Professor Rolf Nevanlinna in dieser Umgebung zu begrüssen, wo er so viele Jahre und ich eine kurze Zeit gewirkt haben. Das heurige Kolloquium ehrt ihn zu seinem achtzigsten Geburtstag. Wir freuen uns alle, dass seine Vitalität es ihm ermöglicht, ohne Spur des Alters in unserer Mitte zu sein.

Der Ruhm unseres Gastes hat viele Gründe. Die Funktionentheoretiker bewundern ihn, weil er besonders eben diese engere Disziplin von Grund aus erneuert hat. Nicht nur die Spezialisten, sondern alle Mathematiker der Welt erkennen ihn als eine der ganz wenigen Spitzenkapazitäten ihrer Wissenschaft an. Die Laien kennen Nevanlinna wegen seiner vielseitigen kulturellen Tätigkeit in seiner Heimat und in der Schweiz und wegen seiner Bemühungen um die Beziehungen zwischen Finnland und der Schweiz.

Das Kuratorium des Rolf Nevanlinna -Kolloquiums hat mich gebeten, meinen Vortrag so zu gestalten, dass auch Nicht-Funktionentheoretiker ihm folgen können. Es ist freilich eine Tatsache, dass Nevanlinnas Produktion zu einem überwiegenden Teil, aber nicht ausschliesslich, auf dem Gebiet der Funktionentheorie liegt, und vor allem ist es sein Einsatz auf diesem Gebiet, der seinen Namen so bekannt gemacht hat, dass der Begriff Nevanlinna-Theorie allen Mathematikern geläufig ist. Trotzdem hoffe ich dem Wunsch des Kuratoriums dadurch entgegenkommen zu können, dass ich hauptsächlich über die Entstehung und Bedeutung der Nevanlinnaschen Ideen spreche, ohne auf technische Einzelheiten einzugehen.

Mein Bestreben wird sein, womöglich diejenigen Punkte in Nevanlinnas Schaffen zu beleuchten, die es bewirkt haben, dass seine Ideen sofort

* Eröffnungsvortrag des achten Rolf Nevanlinna -Kolloquiums in Zürich am 6. November 1975 . 
Anklang gefunden haben und auch auf die Dauer nie an Wert verloren haben. Nicht alle gute Mathematik schlägt sofort an. Ich glaube, vieles hängt von der Persönlichkeit des Mathematikers ab. Solche Züge der Persönlichkeit zeigen sich schon sehr früh, und wenn man nach den charakteristischen Merkmalen sucht, lohnt es sich am besten die Jugendarbeiten genau zu analysieren. Ich meine, dass der Schlüssel zum Erfolg fast immer schon in den frühesten Arbeiten vorhanden ist, wenn auch manchmal verborgen. Die Ideen brauchen Zeit, um reif zu werden, aber im Keim sind sie viel früher da. Ich habe versucht, die ersten Arbeiten von Rolf Nevanlinna, und auch die von seinem Bruder Frithiof, aus diesem Gesichtspunkte aus zu interpretieren. Aus Anlass dieses Vortrages habe ich jetzt diese Arbeiten viel gründlicher gelesen als je zuvor, und es hat sich reichlich gelohnt. Als Schüler von Nevanlinna habe ich in erster Linie seine Theorien durch Vorlesungen und Gespräche gelernt, in zweiter Linie durch seine zusammenfassenden Darstellungen und Monographien. Vielleicht eben deshalb hatte ich versäumt, viele Originalabhandlungen zu lesen, und jetzt fand ich, dass ich von vielen dieser Abhandlungen nicht einmal Sonderdrucke besass. Das alles jetzt nachzuholen, war für mich eine wirkliche Inspiration.

Die frühen Arbeiten von Nevanlinna sind sämtlich funktionentheoretisch, und im Augenblick spreche ich nur von seinen Leistungen in der Funktionentheorie. Um einen besseren Überblick zu erhalten, ist es vorteilhaft, seine diesbezüglichen Arbeiten in drei Kategorien einzuteilen. Erstens, die Arbeiten über Interpolation, und dazu rechne ich auch die Arbeiten über schlichte Funktionen. Zweitens, seine grundlegende Forschung über die allgemeinen Prinzipien der Funktionentheorie, wie etwa die Theorie des harmonischen Masses. Drittens, die Theorie der meromorphen Funktionen, die in ganz besonderem Masse zu dem Ruhm von Nevanlinna beigetragen hat. Diese drei Gebiete lassen sich nicht genau voneinander trennen, sie überlappen sich in gewissen Teilen und befruchten sich gegenseitig.

Nevanlinna debütiert 1919 mit seiner Dissertation Über beschränkte Funktionen die in gegebenen Punkten vorgeschriebene Werte annehmen. Es handelt sich um eine gross angelegte Arbeit über das Interpolationsproblem für beschränkte analytische Funktionen im Einheitskreis, bzw. Funktionen mit positivem Realteil, in keinem Sinne eine Lehrlingsarbeit. Bei der Abfassung dieser Abhandlung hat Nevanlinna gegen bedeutende äussere Schwierigkeiten kämpfen müssen. Die Nachwirkungen des Weltkrieges, der russischen Revolution und besonders des Bürgerkrieges in Finnland waren noch sehr fühlbar und haben sich u. a. darin geäussert, dass die neuere ausländische Fachliteratur fast unerhältlich war. In seiner ausserordentlich wertvollen geschichtlichen Übersicht des Interpolationsproblems ist Nevan- 
linna gezwungen anzugeben, dass er gewisse wichtige Arbeiten nur vom Hörensagen kennt und nie zu Gesicht bekommen hat. Unter diesen Umständen ist es geradezu erstaunlich, dass er doch einen sehr klaren Überblick über den damaligen Stand der Funktionentheorie besitzt, und dass er sich an deren Forschung sofort als ebenbürtiger Teilnehmer engagieren kann.

Das Problem lautet: Es sollen alle in $|z|<1$ analytischen Funktionen $f(z)$ bestimmt werden, die der Bedingung $|f(z)| \leqq 1$ genügen und die in gegebenen Punkten $z_{k}$ vorgeschriebene Werte $w_{k}$ annehmen. Wann ist das Problem lösbar, und wann ist die Lösung eindeutig? Nevanlinna hat nicht gewusst, dass das Problem für endlich viele $z_{k}$ schon von G. Pick gelöst worden war, und zwar in sehr eleganter Weise. Nevanlinna übernimmt eine Methode von I. Schur, die er vereinfacht und weiter entwickelt. Vor allem betrachtet er den Fall unendlich vieler Punkte sowie den Fall, in dem einige oder sogar sämtliche Punkte zusammenrücken. Als Grenzfall findet er in dieser Weise z. B. einen neuen Beweis für den schon bekannten Koeffizientensatz von Carathéodory. Aber die Lösung des Interpolationsproblems mit fast allen Sonderfällen ist nicht das einzige, was den Leser beeindruckt. Zum ersten Mal zeigt Nevanlinna seine souveräne Darstellungskunst, indem er sozusagen zwischen den Zeilen die Berührungspunkte seines Themas mit den allgemeinen Prinzipien der Funktionentheorie entwickelt. Die Rolle des Maximumprinzips in Lindelöfs Fassung, oder fast dasselbe, was man heute Subordination nennt, wird stark betont. Mit Rücksicht auf die späteren Arbeiten von Nevanlinna ist es von gewissem Interesse, dass auch die Jensensche Formel genannt wird, aber bis jetzt nur als Ungleichung und nicht als Gleichung.

Das Interpolationsproblem ist eines von Nevanlinnas Lieblingsgebieten geblieben. Er kommt darauf zurück zuerst schon 1922 mit einer Abhandlung über asymptotische Entwicklungen und das Stieltjessche Momentenproblem. Schliesslich schreibt er 1929 eine durch eine Entdeckung von Denjoy inspirierte endgültige Fassung seiner Interpolationstheorie, wo alle in der Dissertation noch offen gebliebenen Fragen beantwortet werden. Ich will sie nicht näher besprechen, weil sie nur einen indirekten Zusammenhang mit Nevanlinnas übrigen Arbeiten haben.

Zur Interpolation im allgemeinen Sinne zähle ich auch die Theorie der schlichten Funktionen. Durch Koebe wurden die Mathematiker auf ihre Bedeutung aufmerksam gemacht. Als Nevanlinna auf der Arena erschien, hatten schon Bieberbach, und dann auch Faber, die Koeffizientenungleichung $\left|a_{2}\right| \leqq 2$ bewiesen. Damit war auch die Koebesche Konstante 1/4 bekannt, und die von Pick angegebenen Schranken, die von dieser Konstante abhingen, führten zur endgültigen Form des Verzerrungssatzes. Im Jahre 1920 gibt Nevanlinna einen sehr viel einfacheren Beweis für den 
Verzerrungssatz, der u.a. zu der genauen Konvexitätsschranke führt. Alle Funktionentheoretiker kennen diesen Beweis, weil er in dem bekannten Lehrbuch von Bieberbach abgedruckt wurde, leider ohne genaue Quellenangabe. Jedenfalls ist es der jetzt kanonische Beweis des Satzes. Im Jahre 1921 folgt eine ebenso klassisch gewordene Abhandlung über konforme Abbildung von Sterngebieten.

Im Jahre 1922 spricht Nevanlinna meines Wissens zum erstenmal vor einem internationalen Auditorium. Die Gelegenheit ist der fünfte skandinavische Mathematikerkongress in Helsingfors, wo Rolf und sein Bruder Frithiof einander komplettierende Vorträge angekündigt haben. Hier beginnt also die Zusammenarbeit der Brüder, die in der noch im selben Jahr erschienenen berühmten Abhandlung Über die Eigenschaften analytischer Funktionen in der Umgebung einer singulären Stelle oder Linie gegipfelt hat. Es wird wahrscheinlich nie möglich sein, die Einsätze von Rolf und Frithiof genau auseinanderzuhalten. Vermutlich wissen sie selber nicht, was jeder im einzelnen beigetragen hat, und schliesslich ist es vielleicht nicht so wichtig. Aber man darf wohl annehmen, dass die Verteilung des Materials gerade bei diesem Kongress ein Fingerzeig sein soll, wer die Ideen in der späteren gemeinsamen Abhandlung ursprünglich gehabt hat.

Es ist sehr interessant, dass Rolf Nevanlinna seinen Vortrag als "eine Anwendung des Poissonschen Integrals" bezeichnet. Das geschah fast sicher unter dem Einfluss von Lindelöf. Wie alle finnischen Mathematiker war Nevanlinna stark beeinflusst von Lindelöf, und Lindelöf hat immer die Bedeutung der Poissonschen Formel stark betont. Rolf Nevanlinna betrachtet die Poissonsche Formel als einen ganz allgemeinen potentialtheoretischen Satz. Die Anwendung der Potentialtheorie auf analytische Funktionen ist natürlich an und sich nicht neu, aber Nevanlinna verwendet harmonische Funktionen, um möglichst genaue und gleichzeitig genügend einfache Majoranten aufzubauen, und das war eine neue Idee bis auf einen ähnlichen Gedanken bei Carleman, dessen Arbeiten die Nevanlinnas noch nicht kannten. Übrigens sind die Verhandlungen dieses Kongresses voll von wichtigen Beiträgen, darunter Carlemans Arbeit über quasianalytische Funktionen. Man kann vermuten, dass der Kongress einen tiefen Eindruck auf die Brüder Nevanlinna gemacht haben muss, die zu den jüngsten Teilnehmern zählten.

Die Poissonsche Integralformel wird von Rolf Nevanlinna mit der Greenschen Funktion geschrieben und als ein ganz allgemeiner Satz aufgefasst. In einer Halbebene führt er die genaue Poissonsche Formel für einen Halbkreis ein, und leitet aus ihr eine erstaunliche Fülle von neuen Resultaten ab. Unter diesen ist wohl die genaue und wesentlich verallgemeinerte Form des Phragmén-Lindelöfschen Prinzips das wichtigste. Es ist bemerkenswert, dass der Pluslogarithmus $\log ^{+}|f|$, der später fast ein 
Firmenstempel von Nevanlinna wird, hier zum ersten Mal auftritt. Eine zweite Anwendung derselben Methode führt zum Zweikonstantensatz, wenn auch bis jetzt nur für eine Halbebene. Daraus entwickelt sich später die Theorie des harmonischen Masses, die ja zu den allerwichtigsten Schöpfungen Nevanlinnas gehört.

In dem Vortrag von Rolf Nevanlinna wird die Verteilung der Nullstellen nur ganz kurz erwähnt, obzwar er schon früher eine Note darüber veröffentlicht hatte. Als Motivierung gibt er an, dass sein Bruder Frithiof eine bessere Methode besitze, und im Kongressbericht folgt in der Tat die Abhandlung des Bruders, wo die Beziehungen zwischen der Verteilung der Nullstellen und Pole einer meromorphen Funktion in einer Halbebene und dem Anwachsen auf dem Rande behandelt werden. Die Methode, die man also Frithiof Nevanlinna zuschreiben soll, verwendet eine allgemeinere Greensche Formel und erlaubt auch nichtharmonische Vergleichsfunktionen. Es ist wohl ein Zufall, dass hier zum ersten Mal von meromorphen Funktionen geredet wird und dass die entsprechende Verallgemeinerung der Greenschen Formel mit der Jensenschen Formel verglichen wird.

Ich muss jetzt zu der gemeinsamen Abhandlung in Acta Societatis Scientiarum Fennicae zurückkommen. Sie ist, wie gesagt, klassisch geworden. Das liegt nicht nur an den zahlreichen neuen Resultaten, sondern noch mehr an dem Einfluss ihrer Methoden. Ohne Übertreibung lässt sich sagen, dass nach dem Erscheinen dieser Arbeit, oder besser in dem Masse wie sie bekannt wurde, die Funktionentheorie nicht dasselbe wie vorher war. Dem Inhalte nach bringt sie eine Verschärfung und Verallgemeinerung der beiden Kongressvorträge; die Form und der Stil sind unübertreffbar. Von den Resultaten erwähne ich nur folgende: 1) der Zweikonstantensatz, diese ausserordentlich nützliche Verallgemeinerung des Maximumprinzips, 2) die scharfe Fassung des Phragmén-Lindelöfschen Prinzips, 3) Sätze über das identische Verschwinden von analytischen Funktionen, 4) der Satz über beschränktartige Funktionen, der heute eine wichtige Rolle in der Funktionalanalysis spielt. Von diesem Satz, der ja später auf meromorphe Funktionen erweitert wird, sagt Frithiof Nevanlinna an anderer Stelle ausdrücklich, dass er von Rolf herrührt.

Es ist angebracht, an dieser Stelle etwas mehr zu sagen über die Gesamtwirkung von Nevanlinnas mathematischer Tätigkeit auf die Grundlagen und Methoden der Funktionentheorie, ganz abgesehen von den Sätzen, die er bewiesen hat. Man denkt da in erster Linie an die Theorie des harmonischen Masses. Sie ist ein unentbehrliches Hilfsmittel der Funktionentheoretiker geworden. Von dieser einfachen Idee geleitet, ist es möglich geworden, systematisch Majoranten zu berechnen, ohne auf einen glücklichen Zufall warten zu müssen. Das ist ein leitender Gedanke in der grossen Monographie Eindeutige analytische Funktionen, die schnell klassisch 
geworden ist. Bevor ich zu den meromorphen Funktionen komme, will ich noch ein anderes Gebiet berühren, zu dem Nevanlinna einen bedeutenden Beitrag geleistet hat. Ich denke an die Theorie der Riemannschen Flächen. Zum Teil steht seine Leistung in ziemlich engem Zusammenhang mit den meromorphen Funktionen, z. B. wenn er die Riemannschen Flächen mit endlich vielen Windungspunkten klassifiziert und die zugehörigen Abbildungsfunktionen untersucht, übrigens mit sehr klassischen Methoden. Noch wichtiger ist die 1941 erschienene Arbeit über quadratisch integrierbare Differentiale auf offenen Riemannschen Flächen. Diese Arbeit ist der Auftakt zu einer ganzen Reihe von Untersuchungen seiner Schüler und der Schüler seiner Schüler über die Klassifizierung von offenen Riemannschen Flächen.

Jetzt ist es an der Zeit, zu der Theorie der ganzen und meromorphen Funktionen überzugehen. Damit die Nicht-Funktionentheoretiker einen besseren Einblick bekommen können, will ich mir erlauben, zuerst über den Stand dieser Theorie zu berichten um die Zeit, etwa 1923-1925, zu der die einschlägigen Arbeiten Nevanlinnas erscheinen. Von der klassischen Zeit her kannte man die kanonische Produktdarstellung von Weierstrass für die ganzen Funktionen

$$
f(z)=z^{\alpha} e^{g(z)} \prod\left(1-\frac{z}{a_{n}}\right) e^{\frac{z}{a_{n}}+\frac{1}{2}\left(\frac{z}{a_{n}}\right)^{2}+\ldots+\frac{1}{h_{n}}\left(\frac{z}{a_{n}}\right)^{h_{n}}},
$$

wo $a_{n}$ die Nullstellen durchläuft. Wenn alle $h_{n}$ gleich einem festen $h$ gewählt werden können und wenn $g(z)$ ein Polynom ist, sagt man, dass $f(z)$ von endlichem Geschlecht sei. Aus der Produktdarstellung konnte zunächst Hadamard einen wichtigen Zusammenhang zwischen Verteilung der Nullstellen und Wachstumsordnung der Funktion herleiten, etwa so wie die Anzahl der Nullstellen eines Polynoms den Grad und dadurch das Wachstum bestimmt. Von diesem Satze kannte man verschiedene Verfeinerungen, aber es waren meistens Einzelresultate. Diese Sätze waren alle mit ziemlich elementaren Mitteln beweisbar. Ganz anders verhielt es sich mit dem berühmten Satz von Picard, nach dem eine ganze Funktion höchstens einen endlichen Wert auslässt. Von diesem Satze kannte man zunächst nur den ursprünglichen Beweis, in dem die elliptische Modulfunktion die Rolle eines deus ex machina spielt. Der erste sogenannte "elementare" Beweis wurde von E. Borel gegeben, und erst dadurch war der Weg zur Verallgemeinerung des Picardschen Satzes geöffnet. Der Beweis von Borel war reichlich kompliziert, und viele haben sich bemüht, ihn zu vereinfachen. Es war Rolf Nevanlinna vergönnt, den richtigen Schlüssel zu finden, der die Tür zu einem ausserordentlich reichen Kapitel der Funktionentheorie öffnete. Seine Entdeckung führte weit über den 
Picard-Borelschen Gedankenkreis hinaus zu einer erstmaligen systematischen Theorie der meromorphen Funktionen.

Um darüber reden zu können, muss ich an den Hauptinhalt der Nevanlinnaschen Theorie erinnern. Schon lange bekannt war die Jensensche Formel

$$
\log |f(0)|=\frac{1}{2 \pi} \int_{0}^{2 \pi} \log \left|f\left(r e^{i \vartheta}\right)\right| d \vartheta-\log \frac{r^{n}}{\left|a_{1} a_{2} \ldots a_{n}\right|} .
$$

Hier ist $f$ eine für $|z| \leqq r$ reguläre Funktion mit den Nullstellen $a_{1}, \ldots, a_{n}$. Wenn man allgemein die Anzahl der Nullstellen innerhalb des Radius $t$ mit $n(t)$ bezeichnet, so lässt sich das letzte Glied durch

$$
N(r)=\int_{0}^{r} n(t) \frac{d t}{t}
$$

ausdrücken, und man bekommt

$$
\log |f(0)|=-N(r)+\frac{1}{2 \pi} \int_{0}^{2 \pi} \log \left|f\left(r e^{i \vartheta}\right)\right| d \vartheta
$$

Für meromorphe Funktionen erhält man in leichtverständlicher Bezeichnung

$$
\log |f(0)|=N(r, \infty)-N(r, 0)+\frac{1}{2 \pi} \int_{0}^{2 \pi} \log \left|f\left(r e^{i \vartheta}\right)\right| d \vartheta
$$

Nevanlinnas Theorie der meromorphen Funktionen ist in dem Moment geboren, in dem Nevanlinna diese Gleichung auf die Form

$$
\begin{aligned}
N(r, \infty) & +\frac{1}{2 \pi} \int_{0}^{2 \pi} \log \left|f\left(r e^{i \vartheta}\right)\right| d \vartheta \\
& =N(r, 0)+\frac{1}{2 \pi} \int_{0}^{2 \pi} \log \frac{1}{\left|f\left(r e^{i \vartheta}\right)\right|} d \vartheta+\log |f(0)|
\end{aligned}
$$

bringt. In etwas anderer Bezeichnungsweise schreibt er auch

$$
T(r, f)=m(r, f)+N(r, f)=T(r, 1 / f)+O(1)
$$

und allgemeiner

$$
T(r, f)=T(r, 1 /(f-a))+O(1)
$$


Diesen Satz bezeichnet man als den ersten Hauptsatz der Nevanlinnaschen Theorie. Er besagt, dass ein Gleichgewicht besteht zwischen der Anzahl der $a$-Stellen bzw. Pole von $f$ und andererseits der Annäherung an $a$ bzw. $\infty$. Es ist zu bemerken, dass man zu dieser einfachen Formulierung durch Integration der Anzahlfunktion $n(r, a)$ gelangt. Ich schreibe auch den zweiten Hauptsatz hin, um beide Sätze gleichzeitig besprechen zu können. Es gibt zwei Formulierungen, die durch Anwendung des ersten Hauptsatzes auseinander hervorgehen:

$$
\begin{gathered}
\sum_{k=1}^{q} m\left(r, a_{k}\right)<2 T(r, f)-N_{1}(r)+S(r) \\
(q-2) T(r, f)<\sum_{k=1}^{q} N\left(r, a_{k}\right)-N_{1}(r)+S(r) .
\end{gathered}
$$

Hierbei bezeichnet $S(r)$ ein Restglied, das wesentlich kleiner als $T(r)$ ist, und $N_{1}(r)$ ist eine nichtnegative Grösse, deren Bedeutung später besprochen werden soll.

Die Hauptsätze treten nicht sofort in dieser endgültigen Form auf. Man muss bedenken, dass Nevanlinna anfangs nur einen elementaren Beweis des Picardschen Satzes geben wollte. Deshalb betrachtet er in der ersten Note in Comptes Rendus überhaupt nur den Fall einer ganzen Funktion, der erste Hauptsatz kommt überhaupt nicht vor, und eine vorläufige schwache Version des zweiten Hauptsatzes wird ohne Beweis angesagt. Hingegen erscheinen 1924 zwei neue Noten, wo die Theorie viel weiter entwickelt wird. In der ersten wird die charakteristische Funktion $T(r, f)$ definiert und die asymptotische Gleichheit von $T(r, 1 /(f-a))$ und $T(r, 1 /(f-b))$ betont. Weiterhin wird die Ordnung einer meromorphen Funktion durch $\lim \sup _{r \rightarrow \infty} \log T(r) / \log r$ definiert. Damit schuf Nevanlinna eine wirkliche Theorie der meromorphen Funktionen, wo es früher nur Fragmente gab. Er ist sofort in der Lage, den Satz von Hadamard auf meromorphe Funktionen zu erweitern, und allerlei sonstige Konvergenzsätze lassen sich ebenfalls auf meromorphe Funktionen überführen. Alle diese Anwendungen sind äusserst einfach, und man könnte leicht den Eindruck gewinnen, der erste Hauptsatz sei gar nicht tief. In Wirklichkeit verhält es sich umgekehrt. Dass der Satz trotz seiner Einfachheit so spät entdeckt wurde, ist geradezu ein Zeichen, dass es ungewöhnlichen Scharfsinn brauchte, diese Eigenschaft der meromorphen Funktionen zu isolieren, und meiner Ansicht nach ist diese Leistung von Nevanlinna durchaus vergleichbar mit der Bedeutung des zweiten Hauptsatzes für den Picard-Borelschen Ideenkreis.

Die zweite Note von 1924 enthält, ohne Beweis, den Fall $q=3$ des zweiten Hauptsatzes in der Formulierung (II). Als Anwendung formuliert Nevanlinna zwei Sätze, die man als Vorgänger der Defekt- 
relation erkennt. Er sagt: Es gibt höchstens zwei Werte $a$ mit $\lim \sup N(r, a) / T(r)<1 / 3$, und falls $\lim N(r, w) / T(r)=0$ für zwei verschiedene Werte $w$ gilt, so ist $\lim N(r, w) / T(r)=1$ für alle anderen $w$, unter der Bedingung dass man im Falle unendlicher Ordnung gewisse Intervalle von endlicher Gesamtlänge auslässt. Das ist eine Verschärfung des Picardschen Satzes, die von allen früheren Verallgemeinerungen grundverschieden ist.

Nevanlinna ist jetzt zu einem Punkte gelangt, wo er daran denken muss, seine Resultate der ganzen Welt zugänglich zu machen. Das geschieht noch im selben Jahre durch eine grössere Arbeit in Acta Societatis Scientiarum Fennicae unter dem Titel Untersuchungen über den Picard'schen Satz sowie ein paar kleinere Abhandlungen. Im Jahre 1925 erscheint eine fast endgültige Darstellung der ganzen Theorie in Acta Mathematica. Man ist jetzt in der Lage, den vollständigen Beweis des zweiten Hauptsatzes zu verfolgen und die ganz einzigartige Beweisidee zu bewundern. Sie beruht auf einer Eigenschaft der logarithmischen Ableitung, und ich will sie kurz andeuten.

Zunächst erinnere ich an eine Formel, die jetzt unter dem Namen Poisson-Jensen-Nevanlinnasche Formel bekannt ist und wohl zum ersten Mal bei Frithiof Nevanlinna steht. Ich schreibe sie nicht hin, sondern nur die durch Ableitung hervorgehende Formel

$$
\begin{aligned}
\frac{f^{\prime}(z)}{f(z)}= & \frac{1}{2 \pi} \int_{0}^{2 \pi} \log \left|f\left(r e^{i \vartheta}\right)\right| \frac{2 r e^{i \vartheta} d \vartheta}{\left(r e^{i \vartheta}-z\right)^{2}} \\
& +\sum_{\left|a_{\nu}\right|<r} \frac{r^{2}-\left|a_{\nu}\right|^{2}}{\left(z-a_{\nu}\right)\left(r^{2}-\bar{a}_{\nu} z\right)}-\sum_{\left|b_{\nu}\right|<r} \frac{r^{2}-\left|b_{\nu}\right|^{2}}{\left(z-b_{\nu}\right)\left(r^{2}-\bar{b}_{\nu} z\right)} .
\end{aligned}
$$

Das ist nicht eine einfache Formel, und ihre Verwertung ist mit ernsten technischen Schwierigkeiten verbunden. Sie führt jedenfalls zu der Abschätzung

$$
m\left(r, f^{\prime} \mid f\right)=O(\log r T(r))
$$

wo gewisse Ausnahmeintervalle auszuschliessen sind. Man sieht nicht sofort, wie man daraus zu dem zweiten Hauptsatz kommt. Das geschieht durch einen schönen Kunstgriff. Von der Identität

$$
\frac{1}{f-a}+\frac{1}{f-b}=\frac{1}{f} \frac{f}{f^{\prime}}\left(\frac{f^{\prime}}{f-a}+\frac{f^{\prime}}{f-b}\right)
$$

ausgehend, erhält man durch mehrmalige Anwendung des ersten Hauptsatzes bis auf kleinere Restglieder

$$
\begin{aligned}
m(r, a) & +m(r, b)+m(r, \infty) \\
& <2 T(r)-\left(2 N(r, f)-N\left(r, f^{\prime}\right)+N\left(r, 1 / f^{\prime}\right)\right) .
\end{aligned}
$$


Der eingeklammerte Ausdruck, der mit $N_{1}(r)$ bezeichnet wird, is wesentlich positiv, weil er die Anzahl der mehrfachen Stellen misst. Damit ist (II) für $q=3$ bewiesen.

Ursprünglich hat Nevanlinna seinen zweiten Hauptsatz nur für diesen Fall angesagt und bewiesen. Littlewood und Collingwood haben beide sofort gemerkt, dass wesentlich derselbe Beweis für beliebiges $q$ gilt. Man fragt sich unwillkürlich, wie es möglich war, dass das Nevanlinna entgehen konnte. Ich glaube, dass hängt mit einer Erfahrung zusammen, die uns allen geläufig ist. Wenn man sich sehr intensiv mit einer Sache beschäftigt, entwickelt sich oft ein blinder Fleck, und man übersieht leicht das, was anderen sofort ersichtlich ist. Gerade weil er sich vorgenommen hatte, den Satz von Picard zu verschärfen, und gerade weil es ihm so gut gelungen ist, hat er vermutlich gar nicht daran gedacht, dass man noch mehr tun könnte. Es handelte sich um einen so grossen Fortschritt, dass die neuen Gedanken sich überstürzten und sich selber zum Hindernis wurden. Es besteht kein Zweifel, dass der allgemeine Fall unter allen Umständen sehr rasch aufgetaucht wäre, und man kann vermuten, dass er Nevanlinna im Unbewussten vorschwebte. Nevanlinna hat sofort die Wichtigkeit dieser Erweiterung erkannt und hat daraus die berühmte Defektrelation $\sum \delta\left(a_{v}\right) \leqq 2$ hergeleitet. Wenn man auch den Einfluss des Gliedes $N_{1}(r)$ in Betracht zieht, lautet er $\sum(\delta(a)+\vartheta(a)) \leqq 2$, wo $\vartheta(a)$ den sogenannten Verzweigungsindex bezeichnet.

Die Anwendungen des zweiten Hauptsatzes gehen viel weiter als nur bis zu der Defektrelation, und man kann ihn nicht mehr als eine Verallgemeinerung des Picardschen Satzes charakterisieren. Ich erinnere vor allem an die schönen Unitätssätze von Nevanlinna, die einem jeden sofort verständlich sind. Er hat z. B. folgendes bewiesen: Falls es für zwei Funktionen $f_{1}$ und $f_{2}$ fünf verschiedene Werte $a$ gibt, so dass die Wurzeln der Gleichungen $f_{1}=a$ und $f_{2}=a$ zusammenfallen, sogar ohne Berücksichtigung der Vielfachheiten, so sind $f_{1}$ und $f_{2}$ identisch. Merkwürdigerweise ist das bis jetzt ein mehr oder weniger isoliertes Resultat geblieben.

Die Monographie Le théorème de Picard-Borel et la théorie des fonctions méromorphes sowie die relevanten Kapitel in Eindeutige analytische Funktionen sind natürlich die beste Quelle für ein Studium der Nevanlinnaschen Theorie, und das gilt auch für Anfänger. Ich möchte aber ganz besonders seine 1931 erschienenen vier Vorträge empfehlen, die er in Hamburg hielt und die in den Abhandlungen aus dem Mathematischen Seminar der Hamburgischen Universität gedruckt wurden. Es handelt sich um eine Übersicht über den damaligen Stand der Wertverteilungstheorie, und sie ist deshalb wichtig, weil der Verfasser sich erlauben kann, über den Ursprung und die Bedeutung der Wertverteilungslehre zu spekulieren. Hier wird zum ersten Mal die Theorie der rationalen Funktionen als Inspira- 
tionsquelle angegeben, so wie etwa $(1+z / n)^{n}$ zur Exponentialfunktion führt. Von diesem Standpunkt aus liegt es sehr nahe, den zweiten Hauptsatz als einen Grenzfall von der Hurwitzschen Relation anzusehen, welche die Anzahl der Verzweigungspunkte einer geschlossenen Überlagerungsfläche mit deren Geschlecht verbindet. In den Hamburger Vorträgen wird dieser Zusammenhang zwar noch nicht klar ausgesprochen, aber er hat offenbar Nevanlinna vorgeschwebt und kommt ziemlich explizit zum Ausdruck in seinem Vortrag auf dem internationalen Kongress in Zürich 1932. Ich erwähne es, weil gerade diese Seite eine grosse Rolle spielt in der späteren Entwicklung der Theorie.

Es ist Zeit, etwas mehr über diese Entwicklung zu sagen. Es gibt Theorien, die zu einem toten Punkt führen, weil sie ein Thema vollständig ausschöpfen. Bei der Nevanlinnaschen Theorie ist das gar nicht der Fall. Die mathematische Umgebung der Theorie ist so reich und der Boden so fruchtbar, dass neue Wege sich ganz zwanglos eröffnen und zu neuen Einsichten führen. Sehr viele Ideen von Nevanlinna waren nur angedeutet, manchmal vielleicht nur im Unbewussten, aber implizit waren sie vorhanden, und es war seinen Nachfolgern verhältnismässig leicht, diese verborgenen Ideen zu enthüllen. So verhielt es sich mit dem geometrischen Inhalt der Nevanlinnaschen Theorie. Im Jahre 1929 wurde von zwei verschiedenen Seiten, nämlich durch Shimizu in Japan und durch Nevanlinnas Schüler Ahlfors, die Entdeckung gemacht, dass Nevanlinnas charakteristische Funktion eine sehr einfache geometrische Bedeutung zulässt. Wenn man die meromorphe Funktion $f(z)$ als Abbildung auf die Riemannsche Kugel auffasst, und wenn $S(r)$ den Flächeninhalt des Bildes von $|z|<r$ bezeichnet, so zeigt es sich, dass $T(r)=\int^{r} S(r) / r$, oder gleichbedeutend, dass $T(r)$ als Mittelwert von $N(r, a)$ bei veränderlichem $a$ gedeutet werden kann. Diese Tatsache war so leicht zu beweisen, dass man sie fast als trivial ansehen kann, oder höchstens als eine Umdeutung des ersten Hauptsatzes. Jedenfalls ist diese Deutung schon in der Begriffsbildung enthalten, nur durch eine dünne Schicht verschleiert. In einer Rede äussert Carathéodory seine Bewunderung für "die geniale Intuition von Rolf Nevanlinna, der zu einer Zeit, wo die geometrischen Zusammenhänge noch ganz versteckt lagen, nur solche Begriffe entdeckt, ausgebildet und benutzt hat, die später die einfachste geometrische Deutung erfahren sollten".

Die Geometrie kommt auch in anderer Weise zur Geltung. Schon ziemlich früh hatte Frithiof Nevanlinna einen neuen Beweis für den zweiten Hauptsatz gegeben, indem er zu der ursprünglichen Idee von Picard zurückging und die von Poincaré eingeführte Metrik mit konstanter negativer Krümmung verwendete. Das war der erste Schritt in die Richtung der Differentialgeometrie. Später wurde es klar, dass die ganze Theorie 
einen sehr wesentlichen differentialgeometrischen Inhalt besitzt. Aus historischen Gründen kann ich wohl meine eigenen Einsätze nicht ganz überspringen, aber ich erinnere daran, dass ich meinerseits sehr stark von beiden Nevanlinnas beeinflusst war. Aus meinen Arbeiten ging hervor, dass die Differentialgeometrie deshalb eine grosse Rolle spielt, weil man den zweiten Hauptsatz als eine Folge des klassischen Satzes von GaussBonnet herleiten kann. Merkwürdigerweise hat diese Bemerkung, die schon 1937 gemacht wurde, anfangs sehr wenig Aufmerksamkeit erregt, vielleicht weil andere Ereignisse in der Funktionentheorie mehr ins Zentrum getreten waren. Dass der differentialgeometrische Inhalt nicht ganz vernachlässigt wurde, verdankt man vor allem Hermann Weyl und seinem Sohn Joachim. Hermann Weyl war immer sehr begeistert von Nevanlinnas Theorie der meromorphen Funktionen, und hat auch öffentlich seine Bewunderung für diese schöne Schöpfung ausgesprochen. Jetzt, im Jahre 1938, wollte er sich selbst damit beschäftigen, und hat mit seinem Sohn eine Theorie der meromorphen Kurven begründet, d.h. eine Theorie der holomorphen Abbildungen der komplexen Ebene in den projektiven Raum von $n$ komplexen Dimensionen. Es entsteht eine stark geometrisch orientierte Theorie, und es zeigt sich als notwendig und wünschenswert, gleichzeitig auch die in den Grassmannschen Räumen liegenden Begleitkurven zu betrachten. Der erste Entwurf war etwas unvollständig, aber die leitende Idee war durchaus von der Originalität, die man von Weyl erwartet. Etwas später, während des Krieges, wurde dann eine vollständige, aber ziemlich komplizierte, Defektrelation von Ahlfors bewiesen. Zum Schluss veröffentlicht Weyl noch eine zusammenfassende Darstellung, die als die "poetische Version" bekannt geworden ist.

Nun geschieht für etwa 25 Jahre überhaupt nichts in der differentialgeometrischen Richtung; man konnte meinen, die ganze Sache sei tot. Aber dann, in den sechziger Jahren, wird die Theorie von den Differentialgeometern wieder aufgenommen, besonders unter dem Einfluss von S. Chern. In der Zwischenzeit hat sich die Differentialgeometrie in eine Richtung entwickelt, die auch für die Wertverteilungslehre neue Interpretationen gestattet. In einer bekannten Arbeit von R. Bott und Chern wird der erste Hauptsatz auf mehrere Dimensionen und mit Sektionen von Vektorbündeln anstatt Funktionen verallgemeinert. Die Entwicklung gipfelt in den schönen Arbeiten von P. A. Griffiths und seinen vielen Schülern, wo sie erstens sämtliche bekannten Resultate aus der Theorie der meromorphen Kurven einfacher beweisen können und zweitens einen vielversprechenden Zusammenhang mit der algebraischen Geometrie zustande bringen. In diesen Untersuchungen spielt die Idee der negativen Krümmung eine grosse Rolle, und es besteht auch eine enge Beziehung zu den bahnbrechenden Arbeiten von P. Lelong über mehrdimensionale 
Abbildungen, die zeitlich etwas weiter zurückliegen. Auch die Arbeiten von W. Stoll führen sehr weit in die Richtung einer Verallgemeinerung auf mehrere Dimensionen. Die Samen, die vor fünfzig Jahren gesät wurden, haben fruchtbaren Boden gefunden.

Die differentialgeometrische Methode ist eine Weiterentwicklung der Nevanlinnaschen Theorie. Aber es gibt auch eine bedeutende Anzahl von Mathematikern, die sich fortwährend mit der ursprünglichen klassischen Theorie beschäftigt haben. Viele für die Theorie wichtige Fragen haben sich schon früh als schwer angreifbar erwiesen, und sie blieben lange ungelöst. Unter den Mathematikern, die wenigstens zum Teil als direkte Nachfolger von Nevanlinna bezeichnet werden können, indem sie die ursprüngliche Theorie weitergeführt haben, denke ich zunächst an W. Hayman und seine Schüler. Hayman hat nicht nur mehrere offene Fragen endgültig beantwortet, sondern er hat auch eine zusammenfassende Darstellung gegeben, aus der man einè gute Übersicht über die post-Nevanlinnaschen Resultate gewinnt. Von grossem Wert ist auch seine Sammlung von ungelösten Problemen.

In den Vereinigten Staaten ist auch eine neue Nevanlinna-Schule herangewachsen unter der Leitung von A. Edrei und W. Fuchs. Es ist bemerkenswert, dass Edrei ein direkter Schüler von Pólya ist, und nicht von Nevanlinna. Pólyas Einsätze in der Funktionentheorie stammen zum grössten Teil aus der Periode kurz vor Nevanlinna. Sie waren hochinteressant und sehr originell, aber sie wurden leider dann, wenigstens zum Teil, von dem Erfolg der Nevanlinna-Theorie überschattet. Erst viel später hat Edrei gezeigt, wie man gewisse Ideen von Pólya für die Nevanlinnaschen Fragestellungen verwerten kann, und er repräsentiert sozusagen eine Symbiose zwischen Nevanlinna und Pólya. Ganz kürzlich haben diese Methoden einen Gipfel erreicht, indem es A. Baernstein II gelang, eine schöne Vermutung von Edrei zu beweisen, die als "Edrei's spread conjecture" bekannt ist. Es wurde leider zu weit führen, diese Vermutung hier zu präsentieren.

Dagegen möchte ich gern über die neuesten Defektsätze berichten. Es wurde ziemlich früh klar, dass bei ganzen Funktionen die Defektrelation von Nevanlinna, d. h. $\sum \delta(a) \leqq 1$, wenn man $\infty$ nicht zählt, die einzige Bedingung ist. Bei meromorphen Funktionen endlicher Ordnung kennt man weitere Bedingungen. Für solche Funktionen zeigte zunächst Hayman, dass die Summe $\sum \delta(\alpha)^{\alpha}$ für $\alpha>1 / 3$ konvergieren muss, aber nicht notwendig für $\alpha<1 / 3$, und später bewies A. Weitsman die Konvergenz im Grenzfalle $\alpha=1 / 3$. Noch vollständiger sind die Resultate von D. Drasin. Es seien gegeben nicht-negative Zahlen $\delta_{i}$ und $\vartheta_{i}$ mit $0<\delta_{i}+\vartheta_{i} \leqq 1$ und $\sum\left(\delta_{i}+\vartheta_{i}\right) \leqq 2$ sowie beliebige verschiedene komplexe Zahlen $a_{i}$. Dann gibt es eine meromorphe Funktion mit den Defekten 
$\delta\left(a_{i}\right)=\delta_{i}$ und Verzweigungsindizes $\vartheta\left(a_{i}\right)=\vartheta_{i}$, und mit nur diesen. Mit Funktionen endlicher Ordnung geht das nicht immer, aber Drasin zeigt, dass es Lösungen gibt von beliebig schwacher unendlicher Ordnung.

Damit beende ich den Abschnitt über meromorphe Funktionen. Ich will nur nochmals betonen, dass diese Theorie eine ganz aussergewöhnliche Widerstandskraft gezeigt hat gegen allerlei Moderichtungen in der Mathematik, die vielleicht kürzere Wege zum Erfolg bieten. Die NevanlinnaTheorie verlangt viel von denen, die sich mit ihr beschäftigen, aber sie verspricht eine reiche Belohnung.

Weil ich so lange bei den meromorphen Funktionen verweilt habe, könnte man den Eindruck bekommen, dass Nevanlinna sich ausschliesslich mit dieser Frage beschäftigt habe. Das stimmt natürlich nicht, und schon wenn ich von seinen jugendlichen Arbeiten sprach, habe ich ihre Bedeutung für die gesamte Funktionentheorie hervorgehoben. Ganz besonders muss ich nochmals die grosse Monographie Eindeutige analytische Funktionen erwähnen, das klassische Buch, mit dem Generationen von Funktionentheoretikern herangewachsen sind. Hier spricht der reife Mathematiker, der die Kunst versteht, Altes und Neues nebeneinander zu stellen, um die Einheit der leitenden Gedanken zur Geltung kommen zu lassen. Auch in dem Buch Uniformisierung kommt diese Verschmelzung von Klassischem und Modernem sehr deutlich zum Ausdruck.

Aber Nevanlinna ist ein vielseitiger Mathematiker, nicht nur Funktionentheoretiker. In kleinen Ländern wie Finnland muss der Akademiker bereit sein, über die verschiedensten Gebiete seines Faches zu lesen. Nevanlinna tat es nie, ohne sich so gründlich in das betreffende Gebiet zu vertiefen, dass er imstande war, selbständig darüber nachzudenken, ohne zu sehr von der jeweils vorhandenen Literatur beeinflusst zu sein. So kam es, dass er früh ein lebhaftes Interesse für die Grundlagen der Geometrie, für axiomatische Logik und für philosophische Fragen überhaupt entwickelte. Von hier aus kam er dann zunächst zu der Relativitätstheorie, wo er besonders bemüht war, den Inhalt dieser Theorie auch den Laien klarzumachen.

Von der Relativitätstheorie aus ist es nur ein kurzer Schritt zu dem hauptsächlichen Nebeninteresse von Nevanlinna, zu der Differentialgeometrie. Es wäre vielleicht zu erwarten, dass ein Mann wie Nevanlinna, der auf einem Gebiet der Mathematik einen so ungewöhnlich grossen Erfolg gehabt hat, entweder sich damit zufrieden gibt, oder, falls er sich mit anderen Teilen der Mathematik beschäftigen will, dass er das mehr oder weniger zum Zeitvertreib als Liebhaberei betreibt. Aber gar nicht so bei Nevanlinna. Ein ganzes Dezennium, von 1950 bis 1960, sind es Fragen der linearen Algebra und der Differentialgeometrie, die ihn hauptsächlich interessieren. Er macht es sich zur Aufgabe, die grundlegenden Begriffe 
der Differentialgeometrie mit grösster Genauigkeit zu überprüfen. Die klassische Differentialgeometrie war von einem Labyrinth von Bezeichnungen durchzogen. Es hat nicht an Methoden gefehlt, diesen Missstand zu überwinden und man kann vermuten, dass Nevanlinna alle diese Methoden einer kritischen Untersuchung unterworfen hat. Am Ende beschliesst er, seinen eigenen Weg zu gehen, und führt eine koordinatenfreie Bezeichnungsweise ein, die er als absolute Analysis bezeichnet. Mit seinem Bruder schreibt er ein Lehrbuch über dieses Thema und zeigt, dass viele Fragen erheblich an Einfachheit und Klarheit gewinnen. Darüber hinaus hat er auch Arbeiten geschrieben, die sich mit der Differentialgeometrie von indefiniten Metriken und in Hilbertschen Räumen beschäftigen. Meine Kompetenz ist zu gering, um Näheres über diese Arbeiten zu berichten, aber es steht fest, dass sie ein bedeutendes Interesse erregt haben und dass mehrere Schüler von Nevanlinna beachtenswerte Beiträge zur Differentialgeometrie geleistet haben. Seine Ideen sind so persönlich, dass sie zum Teil sogar als Opposition gegen die vorherrschende Richtung in der Differentialgeometrie gedeutet werden können. Es ist wohl zu früh vorauszusagen, welche Dauerwirkung sie haben werden.

Ich hoffe, dass es mir gelungen ist, wenigstens in geringem Grade ein Gefühl für die ganz ungewöhnliche Tiefe der Nevanlinnaschen Ideenwelt hervorzurufen. Ich glaube, die wirkliche Tiefe der Gedanken äussert sich in sehr verschiedener Weise bei verschiedenen Mathematikern. Neulich hat ein bekannter heutiger Mathematiker öffentlich geäussert, er habe sich nie sagen brauchen, er wolle Mathematiker werden, er sei es einfach gewesen. Anderen stehen viele Wege offen, und sie treffen eine Wahl. Bei Nevanlinna glaube ich, dass seine Tiefe philosophisch begründet ist. Er hätte ein grosser Philosoph werden können, und man merkt, dass er philosophisch denkt, aber die mathematischen Genen haben schliesslich überwogen. Dafür sind wir Mathematiker dankbar.

Wir haben uns hier versammelt aus verschiedenen Teilen der Welt, um unseren gemeinsamen Freund Rolf Nevanlinna zu begrüssen und ihm zu danken für alles, was er durch sein Lebenswerk der Wissenschaft geschenkt hat, und für das, was er uns allen persönlich bedeutet hat als Mensch, als Freund, als Lehrer, als Kollege und vor allem als Beispiel. Wir hoffen, dass dieses Kolloquium wieder mal ein Zeugnis von seinem tiefen und dauernden Einfluss auf die Mathematik sein wird.

\author{
Harvard University \\ Department of Mathematics \\ Cambridge, Mass. 02138 \\ USA
}

Eingegangen am 18. November 1975 